'Carrera de Odontología, Facultad de Ciencias de la Salud,

Universidad del Desarrollo, Concepción, Chile.

${ }^{2}$ Departamento de Educación

Médica, Universidad de

Concepción. Concepción, Chile.

${ }^{3}$ Escuela de Medicina sede Viña del Mar, Facultad de Medicina,

Universidad Andrés Bello, Viña del Mar, Chile.

${ }^{4}$ Facultad de Medicina,

Universidad San Sebastián, Concepción, Chile.

${ }^{5}$ Carrera de Medicina, Facultad de

Medicina, Universidad Católica de

la Santísima Concepción, Chile.

${ }^{a}$ Cirujano dentista. Magíster en Educación Médica.

bPsicólogo, Magíster en Psicología.

'Kinesióloga, Magíster en Educación Médica.

¿Magíster en Educación Médica.

eIngeniero Informático, Magister en Educación Médica.

${ }^{f}$ Enfermera matrona. Magíster en Educación Médica.

Trabajo financiado por el proyecto FONDECYT Regular 1121002.

Recibido el 23 de octubre de 2014, aceptado el 12 de mayo de 2015.

Correspondencia a: Paula Edith Gómez Herrera Facultad de Ciencias de la Salud, Universidad del Desarrollo, Sede Concepción. Barros Arana 1375, Concepción Chile.

Teléfono: 412255918 info.pgh@gmail.com

\section{Relación entre el bienestar y el rendimiento académico en alumnos de primer año de medicina}

\author{
PAULA GÓMEZ H. ${ }^{1, a}$, CRISTHIAN PÉREZ V. ${ }^{2, \mathrm{~b}}$, PAULA PARRA P. ${ }^{2, \mathrm{c}}$, \\ LILIANA ORTIZ M. ${ }^{2, \mathrm{~d}}$, OLGA MATUS B. ${ }^{2, \mathrm{e}}$, PETER MCCOLL C. ${ }^{3, \mathrm{~d}}$, \\ GRACIELA TORRES A. ${ }^{4, g}$, ANDREA MEYER K. ${ }^{5, \mathrm{~d}}$
}

\section{Academic achievement, engagement and burnout among first year medical students}

Background: Stress may affect the sense of wellbeing and academic achievement of university students. Aim: To assess the relationship of academic engagement and burnout with academic achievement among first year medical students. Material and Methods: The Utrecht Work Engagement Scale-Student and Maslach Burnout Inventory Student Survey (MBI-SS) were applied to 277 first year medical students of four universities. Their results were correlated with the grades obtained in the different courses. Results: Moderately high engagement and low burnout levels were detected. There was a high level of satisfaction with studies and a moderate exhaustion level. Academic achievement was associated with the degree of engagement with studies but not with burnout. Conglomerate analysis detected a group of students with high levels of wellbeing, characterized by high levels of academic engagement and low burnout. Other group had moderate levels of engagement and lack of personal fulfilment. Other group, identified as extenuated, had high levels of personal exhaustion and depersonalization. Finally the disassociated group had a low academic engagement, low emotional exhaustion, high levels of depersonalization and lack of personal fulfilment. Conclusions: Academic achievement is associated with the level of engagement with studies but not with burnout.

(Rev Med Chile 2015; 143: 930-937)

Key words: Learning; Mental health; Stress, psychological; Students, medical.
I niciar una carrera universitaria puede ser una experiencia positiva, que se asume con motivación e ilusión. Pero, para algunos, puede ser una experiencia estresante de difícil manejo. Al coincidir con los cambios del final de la adolescencia, el acceso a la universidad enfrenta a los jóvenes a numerosos desafíos. El individuo puede experimentar falta de control sobre el nuevo ambiente, potencialmente generador de estrés y, en último término, enfrentar el fracaso académico universitario ${ }^{1}$.

La transición a la universidad requiere que los jóvenes se esfuercen por adaptarse a un ambiente competitivo. El estrés y la ansiedad de los estudiantes suele asociarse a sus expectativas académicas, mayores exigencias de autonomía y a factores sociales, como soledad, problemas financieros y tiempo limitado para la familia y amigos ${ }^{1,2}$.

Los estudiantes pueden sentirse desmotivados, no rendir académicamente o simplemente abandonar sus estudios, con la repercusión social que esto conlleva. Las instituciones de educación superior suelen ser lugares altamente estresantes y cuando este estrés es prolongado o frecuente, puede conducir al síndrome de burnout académico ${ }^{3}$, esto es: estudiantes agotados por las 
exigencias académicas, con una actitud cínica y distante hacia éstas y que se sienten ineficaces. Debido a esto, en la última década son cada vez más frecuentes las investigaciones sobre burnout en las instituciones educativas de nivel superior, para detectar de forma oportuna niveles sintomáticos significativos, posibles indicadores de dificultades futuras en relación al éxito académico o profesional, dando una oportunidad excelente de intervención temprana ${ }^{4}$.

Dado lo relevante del problema, es fundamental que la enseñanza de nivel superior procure el bienestar académico de sus alumnos, de manera que la transición de la educación secundaria a la universitaria sea un proceso de formación integral. En este ámbito surge el concepto de engagement o compromiso académico, como opuesto al burnout, entendido como un estado mental positivo relacionado con los estudios caracterizados por vigor, dedicación y absorción, factor importante en el aprendizaje y desarrollo personal de los estudiantes en la universidad ${ }^{5}$.

El uso del término inglés burnout está ampliamente aceptado en la literatura hispanohablante. Sin embargo, en el caso del engagement, aunque también se usa el término inglés ${ }^{5,6}$, en este artículo se usará su traducción al español: compromiso, de aquí en adelante.

A fin de establecer si la calidad de las experiencias de los estudiantes afecta su desempeño, el presente estudio aborda el burnout y el compromiso académico en estudiantes de Medicina, y su relación con su rendimiento académico, operacionalizado mediante el promedio anual de notas.

\section{Metodología}

\section{Diseño}

Estudio descriptivo, cuantitativo, no experimental transversal y de alcance correlacional.

\section{Participantes}

El estudio se realizó con estudiantes de primer año de Medicina de cuatro universidades privadas chilenas, dos de ellas adscritas al Honorable Consejo de Rectores. Se seleccionó una muestra de 315 estudiantes mediante muestreo no probabilístico por cuotas. Tras eliminar a aquellos con tasas de omisión en los cuestionarios sobre $10 \%$, se obtuvo una muestra válida de 277, descrita en la Tabla 1.

\section{Instrumentos}

Para medir los niveles de compromiso académico se usó el Utrecht Work Engagement Scale-Student de 17 ítems (UWES-SS-17) y para el burnout, el Maslach Burnout Inventory Student Survey (MBI-SS).

El UWES-SS-17 fue elaborado por Schaufeli y cols. para medir el compromiso o engagement en contextos académicos ${ }^{5}$. En universitarios chilenos, fue validado por Parra et al., con la presencia de una estructura bifactorial compuesta por involucramiento en los estudios y satisfacción con los estudios en 17 ítems ${ }^{6}$.

El Maslach Burnout Inventory Student Survey (MBI-SS), creado por Maslach y Jackson en 1981 y validado en población universitaria chilena por Pérez et al., identifica tres factores: agotamiento emocional, despersonalización y falta de realización personal en 22 ítems ${ }^{7}$.

Ambos instrumentos describen conductas, pensamientos y afectos donde el participante debe responder según la frecuencia con que ha experimentado los enunciados de los ítems usando una escala Likert de siete alternativas $(0=$ "ninguna vez/nunca" a 6 = "todos los días/siempre).

Para acceder a los antecedentes académicos de los estudiantes se recurrió a la base de datos académicos de cada carrera, facilitada por cada universidad participante.

\section{Tabla 1. Caracterización de la muestra de los estudiantes de Medicina}

\begin{tabular}{|c|c|}
\hline Variable & Estadísticos descriptivos \\
\hline Edad & $\begin{array}{l}\text { M }=19,21 ; D E=1,25 \\
\text { Mín }=18 ; \text { Máx }=26\end{array}$ \\
\hline Sexo & $\begin{array}{ll}\text { Hombre } & (n=131 ; 47,29 \%) \\
\text { Mujer } & (n=146 ; 52,71 \%)\end{array}$ \\
\hline $\begin{array}{l}\text { Estableci- } \\
\text { miento de } \\
\text { origen }\end{array}$ & $\begin{array}{ll}\text { Municipalizado } & (\mathrm{n}=31 ; 11,19 \%) \\
\text { Particular subvencionado } & (\mathrm{n}=108 ; 38,99 \%) \\
\text { Particular pagado } & (\mathrm{n}=137 ; 49,46 \%) \\
\text { Extranjero } & (\mathrm{n}=1 ; 0,36 \%)\end{array}$ \\
\hline Universidad & $\begin{array}{l}\text { Pertenecientes al CRUCH } \quad(n=114 ; 41,16 \%) \\
\text { No pertenecientes al } \mathrm{CRUCH}(n=163 ; 58,84 \%)\end{array}$ \\
\hline Región & $\begin{array}{ll}\text { Región de Valparaíso } & (\mathrm{n}=35 ; 12,64 \%) \\
\text { Región Metropolitana } & (\mathrm{n}=66 ; 23,82 \%) \\
\text { Región del Bío Bío } \quad(n=176 ; 63,54 \%)\end{array}$ \\
\hline
\end{tabular}

n: Número de observaciones; M: Media aritmética; DE: Desviación estándar; Mín/Máx: Máximo y mínimos observados. 


\section{Procedimiento}

El UWES-S-17 y MBI-SS fueron aplicados al finalizar el primer año de carrera. Las calificaciones se obtuvieron al cerrar el primer año académico. Los resultados de los cuestionarios y los antecedentes académicos fueron pareados con un código asignado a cada estudiante para conservar el anonimato. El uso de la información analizada en este estudio fue autorizada por los estudiantes mediante un procedimiento de consentimiento informado visado por el Comité de Ética de CONICYT.

\section{Análisis de datos}

Se realizó un análisis descriptivo del desempeño académico de los estudiantes y de sus puntuaciones en el UWES-S-17 y MBI-SS. Posteriormente, se calculó la correlación entre éstos mediante el coeficiente de correlación de Pearson.

Para identificar la capacidad predictiva conjunta de los niveles de burnout y compromiso sobre el rendimiento académico, se realizó un modelo de regresión lineal múltiple considerando el promedio ponderado anual (PPA) general estandarizado por escuela como variable dependiente.

Luego se realizó un análisis de conglomerados, que permite identificar perfiles de individuos, aplicando análisis de conglomerado jerárquico aglomerativo para identificar el número de grupos, y análisis de conglomerado no jerárquico para asignar los participantes a éstos.

Para comparar los niveles de bienestar y rendimiento de los conglomerados identificados se empleó ANOVA de un factor.

Se consideró como significativo un $\mathrm{p}<0,05$. El análisis fue realizado en el software STATA SE11.0.

\section{Resultados}

En la Tabla 2 se presenta análisis descriptivo del desempeño académico de los estudiantes, considerando todas las asignaturas cursadas (PPA General) y sólo aquellas aprobadas (PPA Aprobadas). Entre éstas, se decidió trabajar con el PPA General estandarizado por Escuela de Medicina, según la media y desviación estándar específica de cada escuela.

Respecto a su situación académica al finalizar primer año, 267 (96,39\%) estudiantes mantenía su condición de alumno regular y 10 casos habían desertado $(3,61 \%)$.

La Tabla 3 muestra los resultados descriptivos del UWES-S-17 y el MBI-S-S. Ante la ausencia de baremos nacionales, y con fines heurísticos, se transformó todos los puntajes a una escala comparable de 0 a $6(\mathrm{M} / \mathrm{i})$. Así, se observa que los estudiantes presentan un elevado compromiso, con mayor satisfacción que involucramiento con

Tabla 2. Estadísticos descriptivos de promedios ponderados anuales de los estudiantes de Medicina

\begin{tabular}{|lccccc|}
\hline $\begin{array}{l}\text { Promedios } \\
\text { ponderados }\end{array}$ & n & M & DE & Mín & Máx \\
\hline PPA General & 277 & 5,44 & 0,58 & 2,95 & 6,77 \\
\hline PPA Aprobadas & 276 & 5,55 & 0,46 & 4,50 & 6,77 \\
\hline $\begin{array}{l}\text { PPA General } \\
\text { estandarizada }\end{array}$ & 277 & 0,00 & 0,99 & $-4,45$ & 2,02 \\
\hline
\end{tabular}

$\mathrm{N}=277$; n: Número de observaciones; M: Media aritmética; DE: Desviación estándar; Mín/Máx: Máximo y mínimos observados.

\section{Tabla 3. Estadísticos descriptivos de los niveles de compromiso y burnout académico de los estudiantes de Medicina}

\begin{tabular}{|lcrrrrr|}
\hline Factores & $\boldsymbol{\alpha}$ & $\mathbf{M}$ & DE & Mín & Máx & M/i \\
Compromiso académico & & & & & & \\
Involucramiento en los estudios & 0,85 & 50,13 & 13,11 & 11 & 108 & 4,18 \\
Satisfacción con los estudios & 0,79 & 27,30 & 3,98 & 12 & 30 & 5,46 \\
Burnout académico & & & & & & \\
Agotamiento emocional & 0,81 & 19,25 & 7,88 & 1 & 42 & 2,75 \\
Despersonalización & 0,67 & 8,34 & 6,34 & 0 & 35 & 1,19 \\
Falta de realización personal & 0,74 & 11,56 & 6,61 & 0 & 37 & 1,45 \\
\hline
\end{tabular}

$N=277 ; \alpha$ : Alfa de Cronbach; M: Media aritmética; DE: Desviación estándar; Mín/Máx: Máximo y mínimos observados; M/i: Media aritmética dividida por número de ítems del factor. 
los estudios. Además, exhiben bajo burnout, pero un moderado agotamiento emocional.

$\mathrm{Al}$ correlacionar el compromiso y el burnout académico de los estudiantes con su promedio anual de notas del primer año, no se encontraron correlaciones estadísticamente significativas del rendimiento académico estandarizado (PPA General) con el burnout, pero sí de manera directa con el involucramiento en los estudios $(p<0,001)$, de forma que estudiantes más involucrados obtenían mejores calificaciones, Tabla 4.

Para identificar la capacidad predictiva conjunta de los niveles de burnout y compromiso sobre el rendimiento académico, y para identificar su capacidad predictiva específica, se realizó un modelo de regresión lineal múltiple considerando el PPA General estandarizado por escuela como variable dependiente, Tabla 5.

Los resultados mostraron que el compromiso junto al burnout lograban una predicción significativa de 7,49\% del PPA ( $p<0,001)$. Sin embargo, considerando los factores individualmente, sólo el involucramiento con los estudios fue un predictor estadísticamente significativo ( $\mathrm{p}<0,001)$, explicando 7,6\% de la variación del promedio de notas.

Finalmente, para identificar perfiles dentro del alumnado según sus niveles de bienestar (compromiso y burnout académico), se utilizó un análisis de conglomerado jerárquico aglomerativo, empleando el método de Ward, utilizando específicamente la distancia euclídea al cuadrado como medida entre los datos. Este se usó para identificar el número de conglomerados, que según el dendrograma, Figura 1, indicó la presencia de tres grupos de estudiantes según sus resultados en el UWES-S-17 y el MBI-S-S.

Luego, para asignar óptimamente a los estudiantes a cada conglomerado identificado, se

Tabla 4. Correlación de Pearson del compromiso y burnout académico de los estudiantes de Medicina con su promedio ponderado anual

\begin{tabular}{|c|c|c|c|c|c|c|}
\hline Variables & $1^{b}$ & 2 & 3 & 4 & 5 & 6 \\
\hline 1. PPA General estandarizado & - & & & & & \\
\hline 2. Involucramiento en los estudios & $0,27 * * *$ & $0,85^{a}$ & & & & \\
\hline 3. Satisfacción con los estudios & 0,04 & $0,54^{* * *}$ & $0,79^{a}$ & & & \\
\hline 4. Agotamiento emocional & $-0,01$ & $-0,16^{* *}$ & $<0,01$ & $0,81^{\text {a }}$ & & \\
\hline 5. Despersonalización & 0,03 & $-0,13^{*}$ & $-0,21 * * *$ & $0,36^{* * *}$ & $0,67^{\mathrm{a}}$ & \\
\hline 6. Falta de realización personal & $-0,10$ & $-0,52 * * *$ & $-0,43 * * *$ & 0,09 & $0,35^{* * *}$ & $0,74^{a}$ \\
\hline
\end{tabular}

$\mathrm{N}=277 ;{ }^{*} \mathrm{p}<0,05$. 'Alfa de Cronbach; ${ }^{\mathrm{b}}$ Números indican correspondencia con variables de la 1 a la 6 exhibidas en primera columna.

Tabla 5. Resultados de la regresión lineal múltiple de compromiso y burnout académico sobre promedio ponderado anual (PPA) general estandarizado por escuela

\begin{tabular}{|lllll|}
\hline Predictores & B & EE & $\boldsymbol{\beta}$ & $\mathbf{s r}^{\mathbf{2}}$ \\
\hline Constante & $-0,545$ & & & 0,076 \\
\hline Involucramiento en los estudios & $0,028^{* * *}$ & 0,006 & 0,364 & 0,014 \\
\hline Satisfacción con los estudios & $-0,036$ & 0,018 & $-0,145$ & 0,001 \\
\hline Agotamiento emocional & 0,005 & 0,008 & 0,036 & 0,001 \\
\hline Despersonalización & 0,005 & 0,011 & 0,030 & $<0,001$ \\
\hline Falta de realización personal & 0,002 & 0,011 & 0,014 & $<$ \\
\hline
\end{tabular}

$R^{2}=0,09 * * ; R^{2}$ ajustado $=0,07 . \mathrm{N}=277 ;{ }^{*}: \mathrm{p}<0,05 . \mathrm{B}=$ coeficientes de regresión no estandarizados; $\mathrm{EE}=$ errores estándar; $\beta=$ coeficientes de regresión estandarizados; $s r^{2}=$ correlaciones semiparciales al cuadrado; $R^{2}=$ coeficiente de determinación; $R^{2}=$ coeficiente de determinación ajustado. 

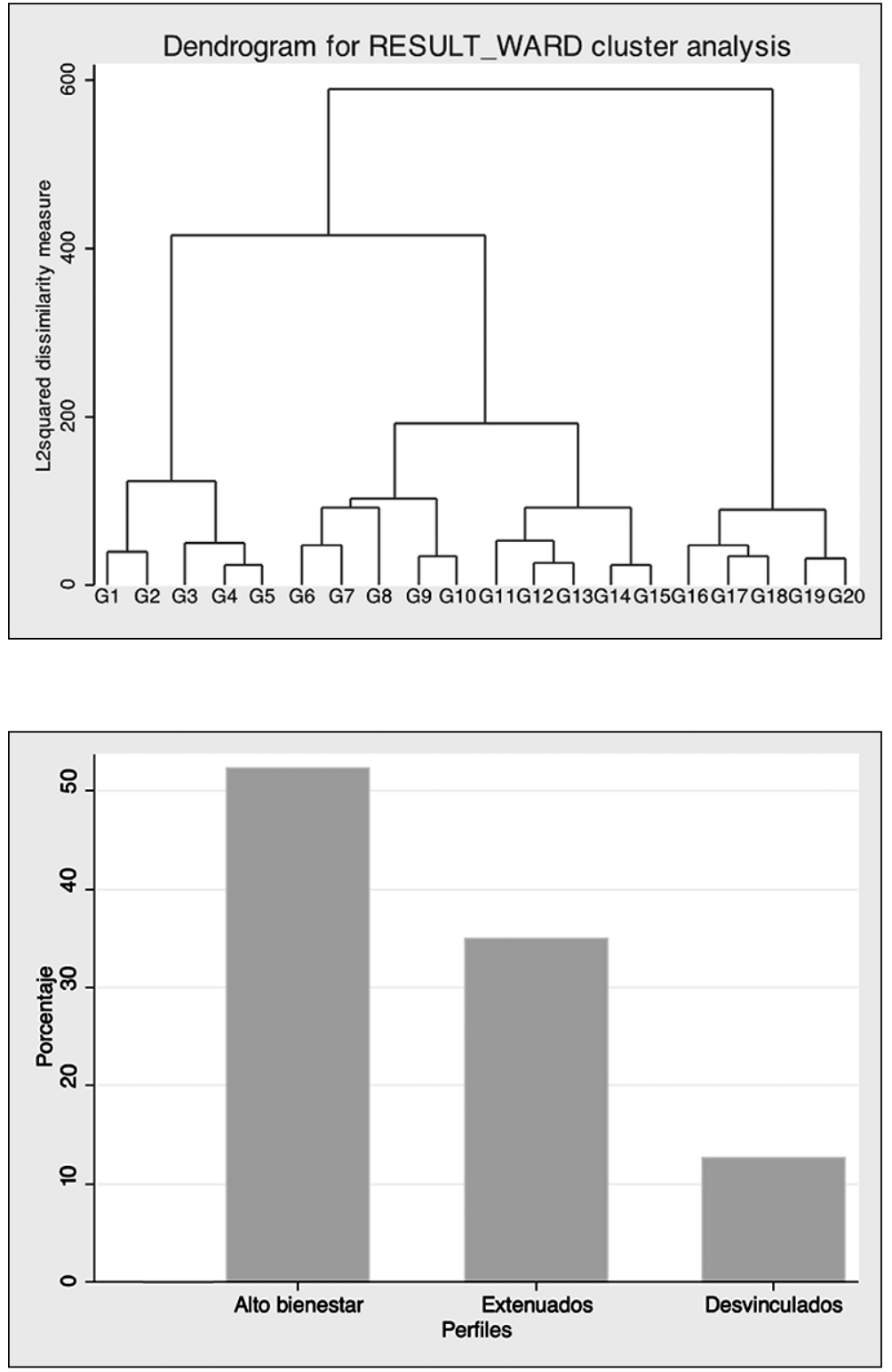

Figura 1. Dendrograma del análisis de conglomerado jerárquico aplicando el método de Ward.
Figura 2. Distribución de los estudiantes en los tres perfiles de bienestar identificados. empleó el análisis de conglomerado no jerárquico, que maximiza la homogeneidad intra-grupos y la heterogeneidad entre grupos. Puntualmente, se aplicó el análisis de K-medias, que asignó a 145 $(52,35 \%)$ estudiantes al primer conglomerado, a $97(35,02 \%)$ al segundo y a $35(12,64 \%)$ al tercero, Figura 2. Los puntajes de las escalas fueron previamente estandarizados.

Aunque el análisis de conglomerado no es una técnica inferencial, se decidió usar ANOVA de un factor, para identificar diferencias estadísticamente significativas en los niveles de compromiso y burnout académico de los tres grupos detectados, Tabla 6.

Los resultados mostraron diferencias estadísticamente significativas entre los conglomerados en los cinco puntajes estudiados. Para identificar diferencias específicas entre los grupos se utilizó la prueba post hoc HSD de Tukey, que mostró en el caso del involucramiento y la satisfacción con 
Tabla 6. Comparación de los puntajes de compromiso y burnout académico para la solución de tres conglomerados del algoritmo K-Medias

\begin{tabular}{|c|c|c|c|c|c|c|c|}
\hline \multirow[b]{2}{*}{ Factores del bienestar } & \multicolumn{2}{|c|}{ Conglomerado 1} & \multicolumn{2}{|c|}{ Conglomerado 2} & \multicolumn{2}{|c|}{ Conglomerado 3} & \multirow[b]{2}{*}{$\mathbf{F}$} \\
\hline & M & DE & M & DE & M & DE & \\
\hline $\begin{array}{l}\text { Compromiso académico } \\
\text { Involucramiento en los estudios } \\
\text { Satisfacción con los estudios }\end{array}$ & $\begin{array}{l}57,16 \uparrow \\
29,05 \uparrow\end{array}$ & $\begin{array}{l}9,46 \\
1,53\end{array}$ & $\begin{array}{l}45,54 \rightarrow \\
27,78 \rightarrow\end{array}$ & $\begin{array}{r}11,10 \\
2,05\end{array}$ & $\begin{array}{l}33,77 \downarrow \\
18,69 \downarrow\end{array}$ & $\begin{array}{r}11,01 \\
4,09\end{array}$ & $\begin{array}{r}88,21 * * * \\
320,66^{* * *}\end{array}$ \\
\hline $\begin{array}{l}\text { Burnout académico } \\
\text { Agotamiento emocional } \\
\text { Despersonalización } \\
\text { Falta de realización personal }\end{array}$ & $\begin{array}{r}15,68 \downarrow \\
5,54 \downarrow \\
8,16 \downarrow\end{array}$ & $\begin{array}{l}6,34 \\
4,27 \\
4,67\end{array}$ & $\begin{array}{l}25,33 \uparrow \\
11,55 \uparrow \\
13,87 \rightarrow\end{array}$ & $\begin{array}{l}6,57 \\
6,56 \\
5,46\end{array}$ & $\begin{array}{l}17,14 \downarrow \\
11,06 \uparrow \\
19,23 \uparrow\end{array}$ & $\begin{array}{l}6,90 \\
7,56 \\
7,34\end{array}$ & $\begin{array}{l}66,23 * * * \\
37,70 * * * \\
74,31 * * *\end{array}$ \\
\hline
\end{tabular}

$\mathrm{N}=277 ;{ }^{*}: p<0,05 ; \uparrow:$ Grupo de puntajes mayores; $\rightarrow$ :Grupo de puntajes intermedios; $\downarrow$ :Grupo de puntajes menores.

Tabla 7. Ilustración simplificada de los niveles de compromiso y burnout para la solución de tres conglomerados

\begin{tabular}{|c|c|c|c|}
\hline & Conglomerado 1 & Conglomerado 2 & Conglomerado 3 \\
\hline $\begin{array}{l}\text { Compromiso académico } \\
\text { Involucramiento en los estudios } \\
\text { Satisfacción con los estudios }\end{array}$ & $\begin{array}{l}\text { Alto } \\
\text { Alto }\end{array}$ & $\begin{array}{l}\text { Medio } \\
\text { Medio }\end{array}$ & $\begin{array}{l}\text { Bajo } \\
\text { Bajo }\end{array}$ \\
\hline $\begin{array}{l}\text { Burnout académico } \\
\text { Agotamiento emocional } \\
\text { Despersonalización } \\
\text { Falta de realización personal }\end{array}$ & $\begin{array}{l}\text { Bajo } \\
\text { Bajo } \\
\text { Bajo }\end{array}$ & $\begin{array}{l}\text { Alto } \\
\text { Alto } \\
\text { Medio }\end{array}$ & $\begin{array}{l}\text { Bajo } \\
\text { Alto } \\
\text { Alto }\end{array}$ \\
\hline
\end{tabular}

*Simplificación de los resultados obtenidos del ANOVA de un Factor de la Tabla 6.

Tabla 8. Comparación del rendimiento académico de los tres perfiles de alumnos de Medicina

\begin{tabular}{|lcccccccc|} 
& \multicolumn{3}{c}{ Alto bienestar } & \multicolumn{2}{c|}{ Extenuados } & \multicolumn{3}{c|}{ Desligados } \\
& M & DE & M & DE & M & DE & F \\
\hline PPA general & 5,51 & 0,59 & 5,39 & 0,56 & 5,33 & 0,60 & 2,03 \\
\hline PPA general estandarizado & 0,06 & 0,97 & $-0,07$ & 1,00 & $-0,05$ & 1,06 & 0,53 \\
\hline
\end{tabular}

$N=277 ;{ }^{*}: p<0,05$.

los estudios, que los tres conglomerados eran estadísticamente diferentes entre sí.

En el caso de agotamiento emocional del MBI-S-S, se encontró que el conglomerado 1 y 3 no diferían significativamente entre sí, pero si lo hacían del conglomerado 2 que presentaba puntuaciones significativamente mayores. En la escala de despersonalización el conglomerado 1 presentaba puntajes significativamente menores que los otros dos, sin diferencias significativas entre estos. Y por último, en la escala de falta de realización personal, se encontró diferencias estadísticamente significativas entre los tres conglomerados. Estos resultados estadísticos se ilustran de manera simplificada en la Tabla 7, en la cual se procedió a conceptualizar las diferencias para etiquetar cada conglomerado.

Así, el primer conglomerado se caracterizó por mayores niveles de compromiso académico y menores niveles de burnout en general, denominándosele grupo de "Alto bienestar". El segundo mostró niveles medios de compromiso académico y falta de realización personal, y mayores niveles de agotamiento emocional y despersonalización, denominándosele grupo de "Extenuados". Y el tercer grupo mostró un bajo compromiso académico 
y agotamiento emocional con un alto nivel de despersonalización y falta de realización personal, denominándosele grupo de los "Desvinculados".

Finalmente, se procedió a comparar el rendimiento de los alumnos de los tres perfiles, pero no se encontró diferencias estadísticamente significativas en el rendimiento académico de ellos, Tabla 8.

\section{Discusión}

El ingreso a la universidad trae cambios abruptos en la mayoría de los jóvenes universitarios, por su exigencia en el ritmo de vida y la metodología de trabajo que emplean los docentes. Hay evidencia de problemas de salud mental en estudiantes de medicina, quienes se beneficiarían, de establecerse programas de intervención orientados al manejo preventivo del estrés ${ }^{8}$. La angustia del estudiante influye en el desarrollo profesional, por lo que las instituciones tienen un interés creciente en promover el bienestar de sus alumnos?.

Uno de los objetivos de este estudio fue describir las calificaciones logradas por los alumnos de medicina en su primer año de estudio. Los estudiantes presentaron altas calificaciones en general, y un bajo porcentaje desertó de la carrera, lo cual puede ser una consecuencia de la exigente selección, dada por el alto puntaje de acceso a la carrera, permitiendo que sea un curso bastante homogéneo en su desempeño ${ }^{5}$.

Por otra parte, se identificaron niveles de bienestar a través del compromiso académico, mostrando niveles moderadamente elevados, siendo mayor la satisfacción que el involucramiento con los estudios, es decir, evalúan positivamente su actividad académica, pero presentan una menor participación efectiva. Esto podría derivar del carácter estresor de la carrera, y las dificultades para adaptar los hábitos de estudio preuniversitarios y conciliar los estudios con otras demandas, como la vida familiar. En relación al burnout académico, se demostraron bajos niveles, sin embargo, la dimensión del agotamiento emocional fue moderado, al igual que lo encontrado en alumnos cubanos de primer año de Medicina, dónde la mayoría de los estudiantes fueron diagnosticados con síndrome de burnout leve ${ }^{10}$.

El burnout y el compromiso son importantes para el éxito y el logro de los objetivos académicos.
Estudios han encontrado que el rendimiento académico se relaciona negativamente con el burnout y positivamente con el compromiso ${ }^{11}$. Valores altos en burnout se relacionan con consecuencias negativas, que se concreta en mal desempeño y bajas expectativas de éxito ${ }^{12}$. En estudiantes españoles, se encontró que el agotamiento, pero no el cinismo, se relaciona de forma negativa $y$ significativa con el rendimiento ${ }^{13}$.

Ahora bien, al relacionar los niveles de compromiso y burnout con el rendimiento académico, en este estudio no hubo correlaciones estadísticamente significativas del rendimiento académico con el burnout. Contrario a lo documentado en otros estudios, el burnout no se asocia al desempeño ${ }^{13}$.

Por otra parte, las calificaciones sí se relacionaron significativamente con el compromiso en su dimensión de involucramiento con los estudios, pero no con la satisfacción con éstos. Esto muestra que más que una buena evaluación de la experiencia en la carrera (p.e, ambiente, prácticas docentes, carga académica, adaptación, etc.), el nivel de esfuerzo constante que el alumno invierte en sus estudios se asocia a un mejor desempeño. Este último resultado coincide con un estudio realizado en universitarios españoles del área de la salud que demostró, que los estudiantes con altos niveles de compromiso académico tenían 1,5 veces más probabilidades de graduarse y requieren aproximadamente 1 semestre menos para hacerlo, obteniendo también un promedio de notas superior. Sin embargo, discrepa con otros hallazgos que han mostrado que el compromiso tiene un valor predictivo sólo para la intención de abandonar los estudios, pero no para el rendimiento académico ${ }^{5}$.

Por último, al intentar identificar los perfiles del alumnado, según sus niveles de compromiso y burnout, se identificaron tres conglomerados: El primero de "Alto bienestar", presenta altos niveles de Compromiso y bajos niveles de burnout, con un comportamiento esperable según la literatura. El segundo grupo, de "Extenuados", presenta niveles medios de compromiso y falta de realización personal y altos niveles de agotamiento emocional y despersonalización, reflejando una experiencia negativa en la carrera, que podría atribuirse a diversos motivos como el ambiente, las prácticas docentes, carga académica, dificultades de adaptación, etc. Sin embargo, presentan una adecuada vinculación afectiva y conductual con 
los estudios, con lo que puede enfrentar la carrera. Finalmente, el tercer grupo de los "Desvinculados" que muestran bajo compromiso y agotamiento emocional con alto nivel de despersonalización y falta de realización personal. Presentan poco compromiso y agotamiento, esa baja vinculación les impide afectarse por sus estudios, por otra parte son cínicos y sienten que lo académico no les reporta satisfacción.

Sin embargo, no se encontraron diferencias estadísticamente significativas en el rendimiento académico de ninguno de estos grupos. Esto muestra que aunque los resultados pueden afectar su salud mental no afecta su desempeño al término del año. Pese a esto, muestra que una décima parte de los estudiantes se sienten "desvinculados" y más de un tercio sobrevive, pese a estar "extenuado". Esto evidencia la necesidad de preocuparse por el bienestar educacional, ya que el profesionalismo médico se encuentra en la actualidad en una etapa de creciente criticismo por el predominio de los intereses personales por sobre el altruismo en los profesionales formados ${ }^{14}$, $y$ en donde un ambiente alienante podría ser el peor enemigo de la formación integral.

Los resultados del estudio muestran que, salvo el involucramiento en los estudios, no existe relación del compromiso y el burnout con el rendimiento académico. No obstante, se evidencia que casi la mitad de los estudiantes muestran un nivel de bienestar académico incompatible con la formación integral de los médicos.

Aún cuando queda pendiente el estudio de los factores predictores de bienestar, es evidente la necesidad de promover el bienestar académico en las escuelas de medicina como indicador clave de una educación de calidad.

\section{Referencias}

1. Pérez C, Bonnefoy C, Cabrera A, Peine S, Macaya K, Baqueano $\mathrm{M}$, et al. Problemas de salud mental en alumnos universitarios de primer año de Concepción, Chile. An Psicol 2012; 28 (3): 797-804.

2. Fouilloux C, Barragán V, Ortiz S, Jaimes A, Urrutia M, Guevara R. Síntomas depresivos y rendimiento escolar en estudiantes de Medicina. Salud Mental 2013; 36: 5965.

3. O’Neill L, Wallstedt B, Eika B, Hartvigsen J. Factors associated with dropout in medical education: a literature review. Med Educ 2011; 45: 440-54.

4. Caballero C, Hederich C, Palacio J. El burnout académico: delimitación del síndrome y factores asociados con su aparición. Rev Latinoam Psicol 2010; 42 (1): 131-46.

5. Casuso-Holgado M, Cuesta A, Moreno N, Labajos M, Barón F, Vega M. The association between academic engagement and achievement in health sciences students. BMC Medical Education 2013; 13: 33.

6. Parra P, Fasce E, Pérez C, Ortiz L, Parra C. Propiedades Psicométricas de la versión extendida del Utrech Work Engagement Scale Student. Rev iberoam diagnost y evaluac psico. En revisión.

7. Pérez C, Parra P, Fasce E, Ortiz L, Bastías N, Bustamante C. Estructura Factorial y Confiabilidad del Inventario de Burnout de Maslach en universitarios chilenos. Rev argent clín psicol 2012; 21 (3): 255-63.

8. Jara D, Velarde H, Gordillo G, Guerra G, León I, Arroyo $\mathrm{C}$, et al. Factores influyentes en el rendimiento académico de estudiantes del primer año de medicina. An Fac Med 2008; 69 (3): 193-7.

9. Dyrbye L, Thomas M, Harper W, Massie S, Power D, Eacker A, et al. The learning environment and medical student burnout: a multicentre study. Med Educ 2009; 43: 274-82.

10. Rosales Y, Rosales F. Burnout estudiantil universitario. Conceptualización y estudio. Salud Mental 2013; 36 (4): 337-45.

11. Schaufeli W, Martínez I, Marques A, Salanova M, Bakker A. Burnout and Engagement In University Students A Cross-National Study. Journal of Cross-Cultural Psychology 2002; 33: 464.

12. Martínez I, Marques A. Burnout en estudiantes universitarios de España y Portugal y su relación con variables académicas. Aletheia 2005; 21: 21.30.

13. Salanova M, Schaufeli W, Martínez I, Breso E. How obstacles and facilitators predict academic performance: the mediating role of study burnout and engagement. Anxiety, Stress, \& Coping 2010; 23 (1); 53-70.

14. Fasce E, Echeverría M, Matus O, Ortiz L, Palacios S, Soto A. Atributos del profesionalismo estimados por estudiantes de medicina y médicos. Análisis mediante el modelo de disponibilidad léxica. Rev Med Chile 2009; 137: 746-52. 